\title{
Research on a Hierarchical and Simultaneous Gravity Unloading Method for Antenna Pointing Mechanism
}

\author{
Guoyong Yang ${ }^{1,2}$, Hongguang Wang ${ }^{1}$, Jizhong Xiao ${ }^{3}$, Zuowei Wang ${ }^{4}$, and Lie Ling ${ }^{1}$ \\ ${ }^{1}$ State Key Laboratory of China, Shenyang Institute of Automation Chinese Academy of Sciences, \\ Shenyang 110016, China \\ ${ }^{2}$ University of Chinese Academy of Sciences, Beijing 100049, China \\ ${ }^{3}$ Department of Electrical Engineering, City College of New York, New York, USA \\ ${ }^{4}$ Beijing Institute of Control Engineering, Beijing, China \\ Correspondence to: Guoyong Yang (ygy2316@163.com)
}

\begin{abstract}
Received: 18 September 2016 - Revised: 14 February 2017 - Accepted: 26 February 2017 - Published: 20 March 2017
\end{abstract}
\begin{abstract}
This paper presents a hierarchical and simultaneous gravity unloading method. An air bearing gravity unloading facility for two-orthogonal-axis antenna pointing mechanism (APM) is designed based on this method. This method is proposed based on the characteristics analysis of the two-orthogonal-axis APM and air bearings. The mechanism of the hierarchical and simultaneous gravity unloading method is described in detail. It solves the coupling problem of two axes and unloads the gravity of both joints hierarchically and simultaneously. The air bearing gravity unloading facility which is a structure mechanism with two layers based on this method is designed with planar air bearing and air spindle. The structure of the facility is described in detail. The dynamic models of the APM with its load in space environment and on the air bearing gravity unloading facility are derived respectively. With the analysing of the driving torques and vertical forces of the APM joints in both models, the results demonstrate that the gravity unloading facility can simulate the microgravity environment successfully. This conclusion is also proved by the dynamic simulation with ADAMS software. The simulation also provides some optimization targets for the gravity unloading facility. At last, the gravity unloading facility is established and some experiments are done. The dynamic models, the simulation results and the experiments all show the effectiveness of the hierarchical and simultaneous gravity unloading method.
\end{abstract}

\section{Introduction}

Tracking and Data Relay Satellite (TDRS) (Zou, 2011; Shaw et al., 2015) is called the satellite of satellites. The TDRS can provide data relay and measurement services for satellites and other spacecraft. It not only is a fundamental solution for the monitoring and control, high coverage communication problems, but also solves the problems of high-speed data transmission and multi-objective monitoring and control. TDRS is usually equipped with one or two large intersatellite link antenna (Zhai and Baoyin, 2008). The antenna is driven by two-axis antenna pointing mechanism (APM) to get its exact direction for the acquisition and tracking of targets. Usually the TDRS's antenna is very large and needs high tracking accuracy (Jinpeng et al., 2006), so there are higher requirements for the pointing mechanism with its capacity of driving performance, pointing accuracy and reliability. It is necessary to test the performance of the antenna pointing mechanism before launching a TDRS to ensure that the APM meets these requirements. So a gravity unloading facility is needed to test the APM on the ground. The facility unloads the gravity of artificial load (which is designed to replace the antenna on the facility) and the APM parts hierarchically and simultaneously to test the performance while the joints of APM are rotating.

Current methods to build a microgravity environment on the ground include the followings (Zhu and Yuan, 2013): microgravity tower (drop tubes) (Sato and Wakabayashi, 2001), air bearing simulator (Hal, 2003), neutral buoyancy simulator (Atkins et al., 2002; Yao and Mei, 2008), suspend- 
ing simulator (Yangsheng et al., 1992; Jagannathan et al., 1995), etc. Among those methods, air bearing simulator is used widely because of its higher level of microgravity, lower cost, simpler structure and wider adaptability. Some countries have established air bearing simulators such as United States (Chernesky, 2001; Schwartz and Hall, 2004; Chung, 2007; Jung and Tsiotras, 2007; Scharf et al., 2010), Japan (Umetani and Yoshida, 1989), Britain (Sandor et al., 2007), Germany (Zhang and Cao, 2006), China (Xu, 2010; Zheng et al., 2010; Qi et al., 2011) and so on. However, most of current simulators are designed for the whole satellite model or robot arms which consist of parallel joints to compensate its gravity and simulate the microgravity environment. Those simulators which based on the overall satellite model can test the dynamic performance of the whole satellite model instead of the performance of the parts inside the satellite model because the gravity effect between the parts is not eliminated by the gravity unload method applied. The gravity unloading facility based on parallel joints robot arms models can only test the performance of certain robot arms with parallel joints.

The APM of TDRS is a two-orthogonal-axis structure and the performance of both joints should be tested, so those gravity unloading facilities above are not applicable. Two joints of APM must be unloaded respectively while they are rotating. The unload componests of rear joint may act as the load of front joint, so there is a coupling problem when designing the gravity unloading facility. The most important thing is that the APM must not be modified to mount the unloading facilities and measure equipment, so the difficulty is increased further. In this paper, a hierarchical and simultaneous gravity unloading method is proposed and many problems are solved for the gravity compensation of two-axis antenna pointing mechanism. These problems includes the gravity unload method, the configuration of the gravity unloading facility, the coupling problem, the design of the artificial load of the antenna, the protection of the APM, the non-contact measurement of joints angular, the measurement of torque and residual gravity torque.

Following the introduction in Sect. 1, Sect. 2 describes the gravity compensation method in detail. In Sect. 3, two dynamic models of the pointing mechanism are derived in space environment and on the gravity unloading facility respectively. The gravity unloading facility is proved to compensate gravity successfully by the comparison of two dynamic models. Section 4 shows the simulation of the air bearing gravity unloading facility with ADAMS software. After that, some experiments are done with the facility built and the last section gives the conclusion.

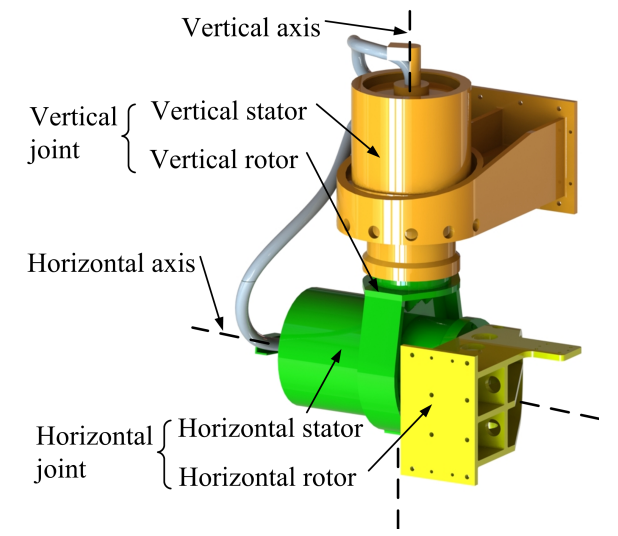

Figure 1. The antenna pointing mechanism.

\section{Air bearing gravity unloading facility for two-orthogonal-axis antenna pointing mechanism}

The antenna pointing mechanism is fixed on the satellite in space to drive the antenna which fixed on the output interface. The antenna pointing mechanism consists of two orthogonal joints, a vertical joint and a horizontal joint as shown in Fig. 1. The vertical joint rotor and the horizontal joint stator are fixed together firmly. Thus the pointing mechanism can be divided into three parts: (1) the vertical stator which is fixed on the satellite, (2) the vertical rotor which is also called the horizontal stator because they are fixed firmly, (3) the horizontal rotor. The antenna is fixed on the output interface of the horizontal rotor. The gravity of three parts and antenna (the antenna is replaced by an artificial load on the gravity unloading facility) should all be compensated to test the driving performance of APM on the ground. What's more, the APM can't support the weight of the antenna in the gravity field on the ground or else it may be destroyed by the gravity of the antenna. Thus, a gravity unloading facility is needed on the ground to test the APM, because one main characteristic of space environment is microgravity. The facility should offer two rotational degrees of freedom (DOFs) for the joints of APM with artificial load mounted.

\subsection{Gravity unload mechanism}

The gravity of two joints of APM and the artificial load must be unloaded and two joints should rotate freely while unloading the gravity. A hierarchical and simultaneous gravity unloading method is proposed.

The artificial load is mounted on the horizontal joint and rotates around the horizontal axis, so an unload facility is need to unload the gravity and offer a rotational DOF around the axis of horizontal joint. Besides, the horizontal joint and artificial load should rotate around the vertical axis together when the vertical joint is rotating. Thus the horizontal joint, the artificial load should all have the vertical rotational DOF 


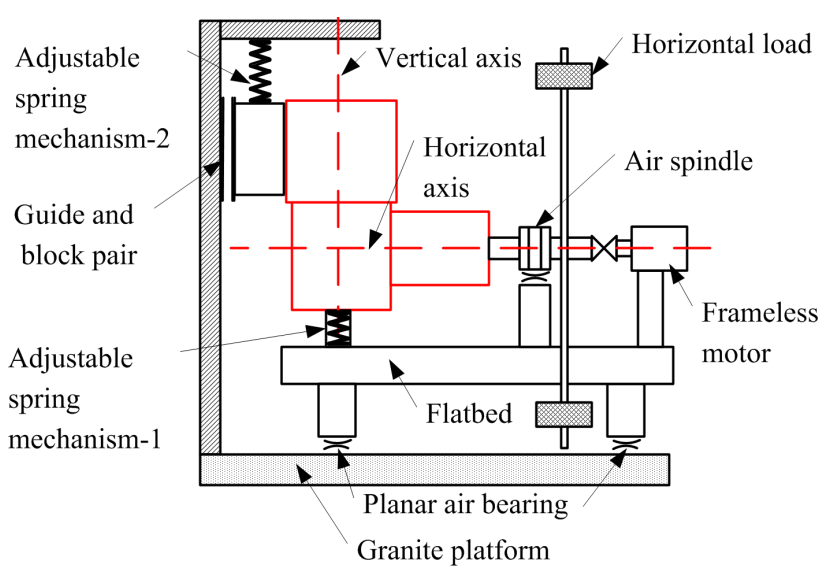

Figure 2. Structure of the gravity unloading facility.

around the vertical axis of the APM while the horizontal joint gravity is unloaded. What's more, the vertical joint gravity should also be unloaded and have the rotational DOF around the vertical axis. So the unloading of the vertical joint gravity and offering vertical rotational DOF can be done by the same component (such as planar air bears or spherical air bearings). The unloading components of horizontal and vertical joint may couple with each other, so the structure must be designed specially and carefully. The APM is designed to run in space, so the APM on the gravity unloading facility cannot support the gravity of the antenna. The facility must offer protection structure to avoid the destruction of the APM by the gravity of artificial load and the unloading parts.

\subsection{Structure of gravity unloading facility}

The gravity must be compensated on the ground to simulate microgravity environment. One method is using air bearings to unload gravity because of its low viscous resistance without direct contact. Currently, there are three kinds of air bearings: planar air bearing, spherical air bearing and air spindle. Their characteristics are shown in Table 1. Planar air bearings and spherical air bearings must be mounted on the horizontal plane while the air spindle can be mounted vertically or horizontally.

The gravity unloading facility for the antenna pointing mechanism should have two DOFs of rotation: a vertical and a horizontal rotation. The whole horizontal joint also rotates around the vertical one when the APM vertical joint is activated.

Both the spherical air bearing and air spindle can be used for the horizontal joint according to the analysis of the pointing mechanism gravity unloading facility. Because only one DOF of rotation is needed, the air spindle is chosen to unload the gravity of horizontal joint taking the structural stability and maintainability. The vertical joint must use several planar air bearings to balance the gravity because the APM and artificial load are not asymmetry around the APM ver-

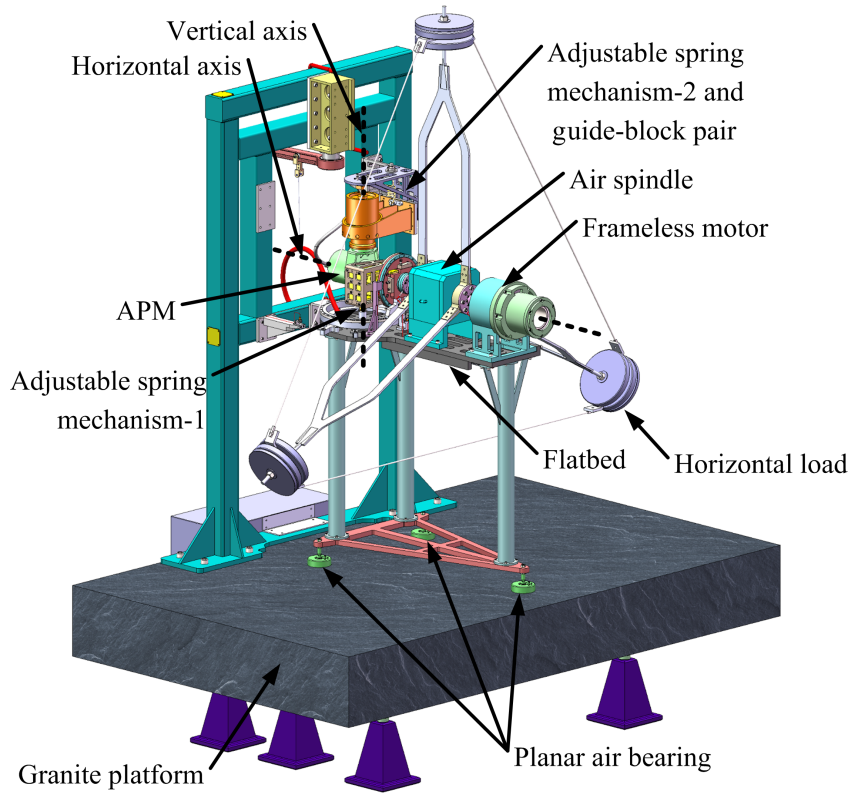

Figure 3. 3-D model of the gravity unloading facility.

tical axis. There are air films in the air bearings, so there is not direct contact between the rotor and stator of the air spindle, so as to the planar air bearings and the granite platform. A frameless motor is chosen also because there is not direct contact between the rotor and stator. The frameless motor is used to offer extra torque which is caused by the cable of the antenna when the TDRS runs in space. With a thick cable, the torque between the APM and the antenna cannot be ignored when the joint of APM is rotating. There is not any cable between the artificial load and the APM on the gravity unloading facility, so the frameless motor is applied to simulate the torque on the facility (shown in Figs. 2 and 3).

The gravity unloading facility consists of the granite platform, a frame on the granite platform to mount the APM, two adjustable spring mechanisms, a guide and block pair, the APM (the red one in Fig. 2), air spindle, planar air bearings, artificial load, frameless motor, the flatbed and other connecting components and measuring devices (shown in Fig. 3). The granite platform supports all the other components through the planar air bearings and the frame on the platform. The APM is fixed on the block of the guide and block pair which is fixed on the frame on the granite platform. Adjustable spring mechanism-2 is fixed between the APM and the frame on the granite platform. The rotor of the air spindle and artificial load is mounted on the horizontal rotor of the APM (on the end interface of the APM). The frameless motor's rotor is fixed on the end of the artificial load while the stator is fixed on the flatbed. Adjustable spring mechanism-1 is mounted between the vertical rotor of APM and the flatbed to support the gravity of the APM vertical joint's rotor. Three planar air bearings are mounted under the 
Table 1. Three kinds of air bearings.

\begin{tabular}{llll}
\hline Characteristics & Planar air bearing & Spherical air bearing & Air spindle \\
\hline DOF & 2 translation, 1 rotation & 3 rotation & 1 rotation \\
Range of motion & On the whole plane & 3 rotational DOFs in one point & Rotate around a fixed axis \\
Precision & Low & Media & High \\
\hline
\end{tabular}

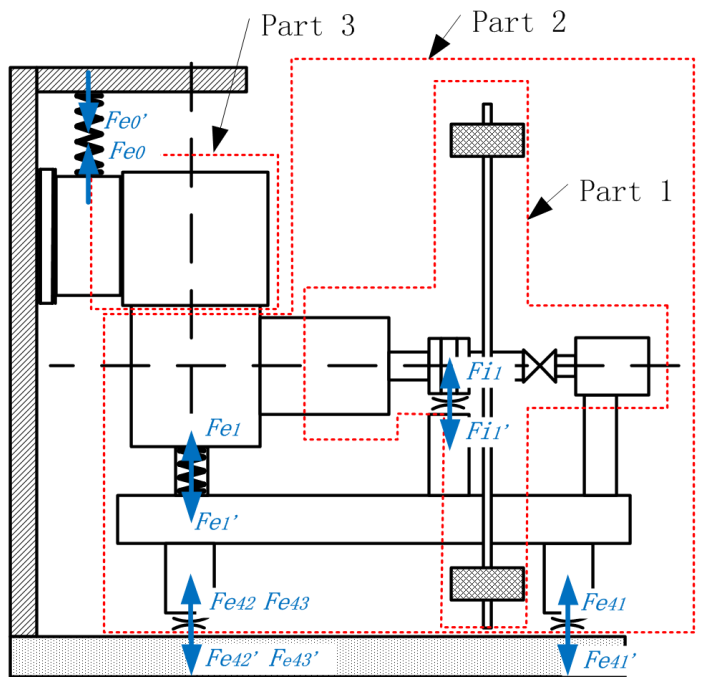

Figure 4. Gravity unloading method.

flatbed and supported by the granite platform via the air films between the air bearings and the platform.

\subsection{The unloading of the gravity}

The gravity unloading facility is designed with two layers (shown in Fig. 4). The bottom layer consists of three planar air bearings placed as a triangle while the upper layer is the air spindle. Three planar air bearings are mounted under the support flatbed while the air spindle is fixed on the top of the flatbed. The facility can be divided into three parts to unload the gravity (shown in Fig. 4).

Firstly, the air spindle bears the gravity of part 1 (including horizontal axis's rotor, the artificial load and the frameless motor's rotor, shown in Fig. 4) by the force $F_{i 1}$ which is offered by the air film inside the air spindle. The gap between the rotor and stator is full of high-pressure air to reduce the friction. The horizontal axis's rotor of APM and the artificial load are mounted on two sides of the air spindle rotor firmly. APM horizontal axis' rotor, the artificial load and other testing parts are all fixed together coaxially with the air spindle rotor and rotate around the horizontal axis. Their gravity is supported by air film in the air spindle without direct contact which has extremely low friction while rotating. Thus the horizontal joint of the APM gets its DOF of rotate around horizontal axis without the influence of gravity.
Secondly, the gravity of horizontal axis's stator and vertical axis's rotor is compensated by the spring mechanism-1 (spring force $F_{\mathrm{e} 1}$ ) fixed on the support flatbed. The centre of gravity of those parts is just within the contact plane pair which is square plane area. There is an adjustment structure to modify the force of the spring to improve the accuracy of compensation. The support flatbed is sustained on the granite platform by three planar air bearings. Three planar air bearings are placed as a triangle, so the gravity centre of part 2 (shown in Fig. 4, including the artificial load, the air spindle, frameless motor, the support flatbed, horizontal axis's stator and vertical axis's rotor, adjustable spring mechanism-1) is just above the centroid of the triangle. This makes the load of three planar air bearings relatively even. The gap between the planar air bearings and the granite is also full of high pressure air which supports the flatbed by the force $F_{\mathrm{e} 41}, F_{\mathrm{e} 42}$ and $F_{\mathrm{e} 43}$. Thus part 2 can rotate around the vertical axis only with air viscous resistance of three planar air bearings which is extremely small. What's more, the air spindle stator is also fixed on the support flatbed and rotates around the vertical axis together with the flatbed driven by the vertical joint. So the horizontal and the vertical joint rotate together without any interference as if there is no gravity.

Thirdly, the gravity of part 3 (vertical axis's stator, shown in Fig. 4) is unloaded precisely by adjustable spring mechanism-2 (a spring which is also connected to an adjustable structure). The adjustable structure can offer the force $F_{\mathrm{e} 0}$ which is equal to the weight of part 3. Guide and block pairs are used to set the vertical translation DOF of part 3 free because there are vertical movements of the planar air bearings and air spindle while the start and end of the simulation. The spring and guide-block pairs mechanism fixed on the granite platform frame can protect the APM from being damaged by the extra force of air bearings. With only one DOF of vertical translation in extremely small range, part 3 still acts as a base of part 2 and part 1 .

The gravity unloading facility offers two DOFs of rotation around vertical and horizontal axes with the gravity compensation method described above. Especially, the unloading is working no matter these two axes rotate one after another or together. Three planar air bearings are placed on the same plane to ensure the rotation around vertical axis while unloading part 2. The air spindle can still unload part 1 while it is rotating around horizontal axis. With air films, both axes rotate with extremely low air viscous resistance. Thus the 


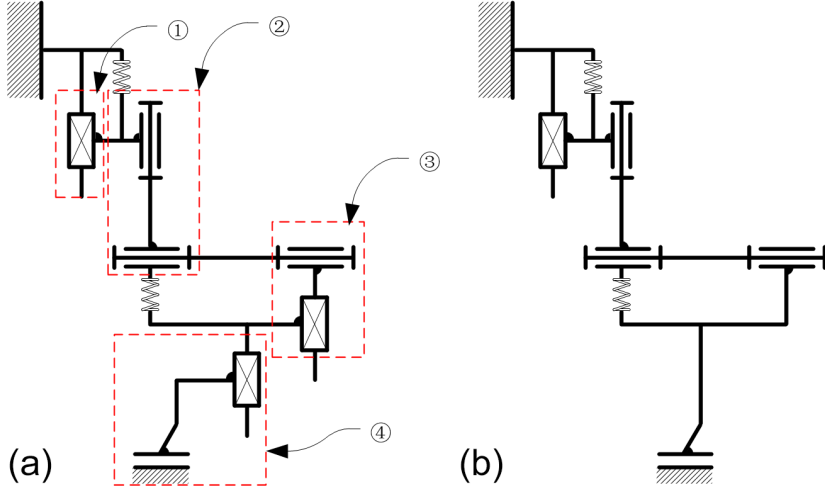

Figure 5. Schematic diagram of gravity unloading facility.

APM runs in the environment of microgravity just as running in space.

\subsection{Running state of the gravity unloading facility}

As shown in Fig. 5a, part 1 is the guide and block pair to set free of vertical movement, part 2 is the APM, part 3 is the air spindle while part 4 is the planar air bearings. During the initialization process of the gravity unloading facility, the balance of the air films and the gravity is accomplished with vertical movement in extremely small range, so there are slider joints in the air spindle and planar air bearings.

There are three processes during the performance test of the APM: the initialization process after pressing the start button, the testing process and the ending process when the test is over. During the initialization process, the schematic diagram is shown in Fig. 5a. The air spindle and planar air bearings all move upward while the slider joint in part 1 is also move upward. The air films in the air spindle and planar air bearings are accomplished with high stiffness. Then during the testing process, the slider joints in air spindle and air bearings do not move at all with stable high pressure air supply. There is not any upward movement, so those slider joints can be replaced by firmly fixed connection shown in Fig. 5b. The slider joint in part 1 is not replaced just to protect the APM from being damaged by extra force. After the test, the high pressure air supply is cut off. The schematic diagram is changed back to the structure shown in Fig. 5a. The slider joints in parts 1, 3, 4 all move downward to eliminate the air films in air spindle and planar air bearings.

\section{Dynamic models}

Two dynamic models of the APM with artificial load in space environment and on the gravity unloading facility are built respectively. They are compared to verify the effectiveness of the facility mentioned above. Air bearings are used to balance the gravity on the facility, so the dynamic model is also changed with the change of mass distribution. There are also

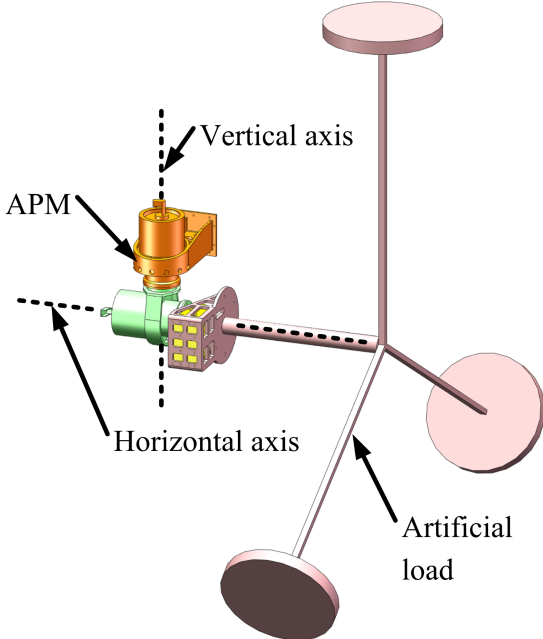

Figure 6. Antenna pointing mechanism in space.

Table 2. Link parameters of the model in space.

\begin{tabular}{lrrrr}
\hline No. & $\alpha_{i-1}$ & $a_{i-1}$ & $d_{i}$ & $\theta_{i}$ \\
\hline 1 & 0 & 0 & $l_{1}$ & $\theta_{1}$ \\
2 & $90^{\circ}$ & 0 & 0 & $\theta_{2}$ \\
\hline
\end{tabular}

some extra unloading forces on the gravity unloading facility when it is working, so the dynamic model with the unloading force must be derived specially. Newton-Euler is used to derive the dynamic models taking the extra unloading forces into consideration. So both dynamic models are derived with Newton-Euler method. The torque and vertical force of APM links are chosen to proof the effectiveness of the gravity unloading method. This is because that the torque is the output of the APM to drive the antenna and the vertical force is influenced by the gravity. The gravity unloading method is effective if the torque and vertical force of APM links are the same between the models in space and on the facility.

\subsection{Dynamic model in space environment}

The gravity unloading facility is designed to test the performance of the antenna pointing mechanism, so the interaction between the pointing mechanism and the satellite is ignored. The APM is mounted on a fixed base. The model of pointing mechanism in space environment is shown in Figs. 6 and 7 with its coordinates and parameters. No gravity acceleration is considered in space.

The vertical joint's stator is the base (link 0). The vertical joint's rotor and horizontal joint's stator are link 1 while the horizontal joint's rotor and the artificial load are link 2.

The D-H coordinate system is shown in Fig. 7. The kinematic model is derived with parameters in Table 2 . 

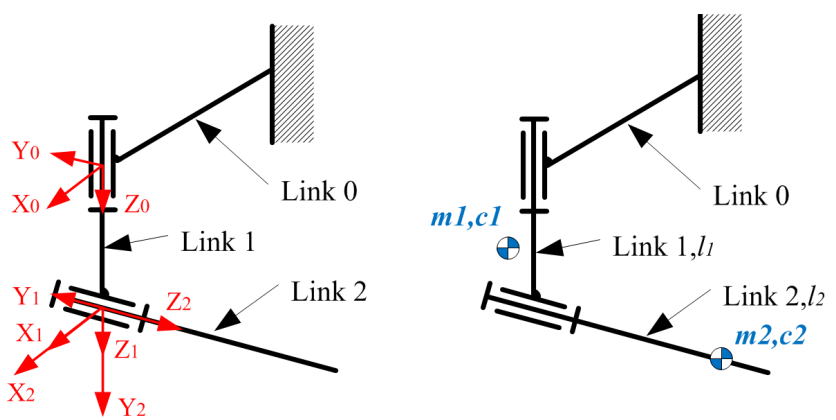

Figure 7. Kinematic sketch with D-H coordinate system.

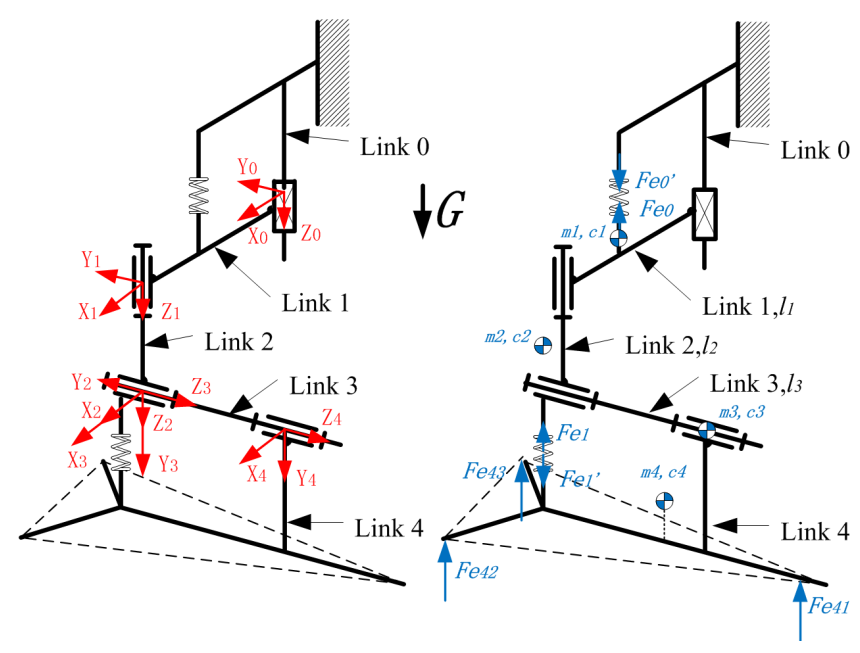

Figure 8. Antenna pointing mechanism on the facility.

${ }_{2}^{0} \boldsymbol{T}={ }_{1}^{0} \boldsymbol{T}_{2}^{1} \boldsymbol{T}$

where

${ }_{i}^{i-1} \boldsymbol{T}=\left[\begin{array}{cccc}c \theta_{i} & -s \theta_{i} & 0 & a_{i-1} \\ s \theta_{i} c \alpha_{i-1} & c \theta_{i} c \alpha_{i-1} & -s \alpha_{i-1} & -s \alpha_{i-1} d_{i} \\ s \theta_{i} s \alpha_{i-1} & c \theta_{i} s \alpha_{i-1} & -c \alpha_{i-1} & c \alpha_{i-1} d \\ 0 & 0 & 0 & 1\end{array}\right]$,

$\theta_{i}$ is the angle of joint $i, s \theta_{i}$ and $c \theta_{i}$ represent $\sin \theta_{i}$ and $\cos \theta_{i}$ respectively.

The dynamic model is derived with Newton-Euler method (shown in Appendix A).

The followings are the driving torque and vertical force of joint 1 and joint 2 in model without gravity:

$$
\begin{aligned}
\tau_{1} & =I_{x x 2}\left(\ddot{\theta}_{1} s^{2} \theta_{2}+2 \dot{\theta}_{1} \dot{\theta}_{2} s \theta_{2} c \theta_{2}\right) \\
& +I_{y y 2}\left(\ddot{\theta}_{1} c^{2} \theta_{2}-2 \dot{\theta}_{1} \dot{\theta}_{2} s \theta_{2} c \theta_{2}\right) \\
& +I_{z z 1} \ddot{\theta}_{1}+m_{2} \ddot{\theta}_{1} l_{\mathrm{c} 2}^{2}+m_{1} \ddot{\theta}_{1} l_{\mathrm{c} 1 y}^{2}
\end{aligned}
$$

Table 3. Link parameters of the model on the facility.

\begin{tabular}{lrrrr}
\hline No. & $\alpha_{i-1}$ & $a_{i-1}$ & $d_{i}$ & $\theta_{i}$ \\
\hline 1 & 0 & $l_{1}$ & $d_{1}$ & 0 \\
2 & 0 & 0 & $l_{2}$ & $\theta_{2}$ \\
3 & $90^{\circ}$ & 0 & 0 & $\theta_{3}$ \\
4 & 0 & 0 & $l_{3}$ & $-\theta_{3}$ \\
\hline
\end{tabular}

$f_{1 \mathrm{~V}}={ }^{1} \boldsymbol{f}_{1} \hat{\mathbf{z}}=0$

$\tau_{2}=I_{z z 2} \ddot{\theta}_{2}+\left(I_{y y 2}-I_{x x 2}\right) \dot{\theta}_{1}^{2} s \theta_{2} c \theta_{2}$

$f_{2 \mathrm{~V}}={ }^{2} \boldsymbol{f}_{2} \hat{\mathbf{X}} \sin \theta_{2}+{ }^{2} \boldsymbol{f}_{2} \hat{\mathbf{Y}} \cos \theta_{2}=0$

Where $\tau_{i}$ is the torque of joint $i, f_{i \mathrm{~V}}$ is the vertical force of joint $i, \dot{\theta}_{i}$ is the angle velocity of joint $i, \ddot{\theta}_{i}$ is the angle acceleration of joint $i, I_{x x i}, I_{y y i}$ and $I_{z z i}$ are the elements of inertia matrix of link $i,\left(0, l_{\mathrm{c} 1 y},-l_{\mathrm{c} 1 z}\right)^{\mathrm{T}}$ is the gravity center position of link 1 in $O X_{1} Y_{1} Z_{1},\left(0,0, l_{\mathrm{c} 2}\right)^{\mathrm{T}}$ is the gravity center position of link 2 in $O X_{2} Y_{2} Z_{2},{ }^{i} f_{i}$ is the force of joint $i$ which is shown in Appendix B. $m_{i}$ is the mass of link $i$, $I_{x x 2}=I_{y y 2}$ with the symmetrical structure.

\subsection{Dynamic model on the gravity unloading facility}

The APM don't move in the vertical direction on the guide and block pairs when the gravity unloading facility running stably. But the slider joint in part 1 (shown in Fig. 5) is maintained just to protect the APM from being damaged by extra force. On the facility, air spindle is used to compensate the gravity of part 3 (shown in Fig. 4). It is considered as a third rotational joint when deriving the dynamic model, shown in Figs. $5 \mathrm{~b}$ and 8 . The horizontal joint's rotor, air spindle rotor, the artificial load and frameless motor's rotor are link 3 while air spindle stator, frameless motor's stator, support flatbed and planar air bearings are link 4 . The balancing state of link 1 , link 2 and link 4 is changed to unload the gravity of part 3 and part 2. There are extra forces: $\boldsymbol{F}_{\mathrm{e} 0}, \boldsymbol{F}_{\mathrm{e} 0}^{\prime}, \boldsymbol{F}_{\mathrm{e} 1}$, $\boldsymbol{F}_{\mathrm{e} 1}^{\prime}, \boldsymbol{F}_{\mathrm{e} 41}, \boldsymbol{F}_{\mathrm{e} 42}$ and $\boldsymbol{F}_{\mathrm{e} 43}$. Where, $\boldsymbol{F}_{\mathrm{e} 41}, \boldsymbol{F}_{\mathrm{e} 42}$ and $\boldsymbol{F}_{\mathrm{e} 43}$ are the unloading forces of planar air bearings acting on link 4 . $\boldsymbol{F}_{\mathrm{e} 1}$ and $\boldsymbol{F}_{\mathrm{e} 1}^{\prime}$ are the interaction force of spring mechanism-1 between link 2 and link 4 to unload the gravity of horizontal joint's stator and vertical joint's rotor. $\boldsymbol{F}_{\mathrm{e} 0}$ and $\boldsymbol{F}_{\mathrm{e} 0}^{\prime}$ are the interaction force of spring mechanism-2 between link 1 and the frame mounted on the granite platform.

The D-H coordinate system is shown in Fig. 8. The kinematic model is derived with parameters in Table 3.

${ }_{2}^{0} \boldsymbol{T}={ }_{1}^{0} \boldsymbol{T}_{2}^{1} \boldsymbol{T}_{3}^{2} \boldsymbol{T}_{4}^{3} \boldsymbol{T}$

The dynamic model equation during the derivation is changed with the extra force mentioned above, so the equation in Newton-Euler is also changed. Taking link 2 as an 
example, there is an extra force $\boldsymbol{F}_{\mathrm{e} 1}$. During the derivation, the force and torque formulation are as follows:

$$
\begin{aligned}
{ }^{2} \boldsymbol{f}_{2} & ={ }_{3}^{2} \mathbf{R}^{3} \boldsymbol{f}_{3}+{ }^{2} \boldsymbol{F}_{2}-{ }^{2} \boldsymbol{F}_{\mathrm{e} 1} \\
{ }^{2} \boldsymbol{n}_{2} & ={ }^{2} \boldsymbol{N}_{2}+{ }_{3}^{2} \mathbf{R}^{3} \boldsymbol{n}_{3}+{ }^{2} \boldsymbol{P}_{\mathrm{c} 2} \times{ }^{2} \boldsymbol{F}_{2}+{ }^{2} \boldsymbol{P}_{3} \times{ }_{3}^{2} \mathbf{R}^{3} \boldsymbol{f}_{3} \\
& +{ }^{\mathrm{c} 2} \boldsymbol{P}_{F_{\mathrm{e} 1}} \times{ }^{2} \boldsymbol{F}_{\mathrm{e} 1}
\end{aligned}
$$

Where ${ }^{i} \boldsymbol{f}_{i}$ and ${ }^{i} \boldsymbol{n}_{i}$ are the force and torque of joint $i \cdot{ }_{i+1}^{i} \mathbf{R}$ is the transformation matrix from coordinate $i+1$ to coordinate $i .{ }^{2} \boldsymbol{F}_{\mathrm{e} 1}$ is the unloading force of part $2,{ }^{\mathrm{c} 2} \boldsymbol{P}_{F_{\mathrm{e} 1}}$ is the position vector of unloading force in coordinate of c2. ${ }^{i} \boldsymbol{F}_{i}$ and ${ }^{i} \boldsymbol{N}_{i}$ are the inertial force and torque of link $i .{ }^{i} P_{\mathrm{c} i}$ is the gravity center of link $i$ in coordinate $i .{ }^{i} P_{i+1}$ is the origin position of coordinate $i+1$ in coordinate $i$.

The force and torque formulations of link 1 and link 4 are also changed with extra force $F_{\mathrm{e} 0}, F_{\mathrm{e} 1}^{\prime}, F_{\mathrm{e} 41}, F_{\mathrm{e} 42}$ and $F_{\mathrm{e} 43}$ just as link 2 .

Thus the followings are torques of three joints:

$$
\begin{aligned}
& \tau_{2}^{\prime}=m_{4} \ddot{\theta}_{2} l_{3}^{2}-2 m_{4} l_{3} l_{\mathrm{c} 4 z} \ddot{\theta}_{2}+m_{2} \ddot{\theta}_{2} l_{\mathrm{c} 2 y}^{2}+m_{3} \ddot{\theta}_{2} l_{\mathrm{c} 3 z}^{2} \\
& +m_{4} \ddot{\theta}_{2} l_{\mathrm{c} 4 z}^{2}+I_{y y 3} \ddot{\theta}_{2}+I_{z z 2} \ddot{\theta}_{2}\left(I_{x x 3}-I_{y y 3}\right) \ddot{\theta}_{2} s^{2} \theta_{3} \\
& +I_{y y 4} \ddot{\theta}_{2}+2\left(I_{x x 3}-I_{y y 3}\right) \dot{\theta}_{2} \dot{\theta}_{3} s \theta_{3} c \theta_{3} \\
& f_{2 \mathrm{~V}}^{\prime}={ }^{2} \boldsymbol{f}_{2}^{\prime} \hat{\mathbf{Z}}=\left[\begin{array}{l}
F_{\mathrm{e} 41}+F_{\mathrm{e} 42}+F_{\mathrm{e} 43} \\
+\left(m_{2}+m_{3}+m_{4}\right)\left(\ddot{d}_{1}-g\right)
\end{array}\right]=0 \\
& \tau_{3}^{\prime}=I_{z z 3} \ddot{\theta}_{3}-F_{\mathrm{e} 42} l_{F_{\mathrm{e} 42} x}-m_{4} l_{\mathrm{c} 4 y}\left(l_{3}-l_{\mathrm{c} 4 z}\right) \ddot{\theta}_{2} \\
& +F_{\mathrm{e} 43} l_{F_{\mathrm{e} 42} x}+\left(-I_{x x 3}+I_{y y 3}\right) \dot{\theta}_{2}^{2} s \theta_{3} c \theta_{3} \\
& f_{3 \mathrm{~V}}^{\prime}={ }^{3} \boldsymbol{f}_{3}^{\prime} \hat{\mathbf{X}} \sin \theta_{3}+{ }^{3} \boldsymbol{f}_{3}^{\prime} \hat{\mathbf{Y}} \cos \theta_{3} \\
& =\left[\begin{array}{l}
F_{\mathrm{e} 41}+F_{\mathrm{e} 42}+F_{\mathrm{e} 43}-k_{2} \Delta x_{2} \\
+\left(m_{3}+m_{4}\right)\left(\ddot{d}_{1}-g\right)
\end{array}\right]=0 \\
& \tau_{4}^{\prime}=\left(F_{\mathrm{e} 43}-F_{\mathrm{e} 42}\right) l_{F_{\mathrm{e} 42 x}}-m_{4} l_{\mathrm{c} 4 y}\left(l_{3}-l_{\mathrm{c} 4 z}\right) \ddot{\theta}_{2}
\end{aligned}
$$

Where $\tau_{i}^{\prime}$ and ${ }^{i} \boldsymbol{f}_{i}^{\prime}$ is the torque and force of joint $i$ on the facility. $f_{i \mathrm{~V}}^{\prime}$ is the vertical force of joint $i . m_{i}$ is the mass of link $i$ while $\left(0, l_{\text {ciy }},-l_{\mathrm{c} i z}\right)^{\mathrm{T}}$ is the gravity center of link $i$ in coordinate $i . I_{x x i}, I_{y y i}, I_{z z i}$ is the element of inertia matrix of link $i$. With the symmetrical structure, $I_{x x 3}=I_{y y 3} . \ddot{d}_{1}$ is the acceleration of translational joint $1 . g$ is the gravity acceleration. $\left(l_{\mathrm{e}_{\mathrm{e} 2} x}, l_{4},-l_{F_{\mathrm{e} 42} z}\right)^{\mathrm{T}}$ is the action point of extra force $F_{\mathrm{e} 42}$ in coordinate $4 . k_{2}$ is the stiffness of the adjustable spring mechanism- 1 while the $\Delta x_{2}$ is the deformation of the spring. Joint 1 is static when the facility is running stable, so $\ddot{d}_{1}$ is 0 . Besides, three planar air bearings support $m_{2}, m_{3}$ and $m_{4}$ while adjustable spring mechanism- 1 supports $m_{2}$. So $F_{\mathrm{e} 41}+F_{\mathrm{e} 42}+F_{\mathrm{e} 43}=\left(m_{2}+m_{3}+m_{4}\right) g$ while $m_{2} g=k_{2} \Delta x_{2}$.

The last joint (joint 4 ) is the air spindle which is a passive joint, so the gravity and the torque of three planar air bearings act as its driving torque. The driving torque of joint 4 is neglected. The torque of joint 3 is also changed as follows:

$$
\begin{aligned}
& \tau_{4}^{\prime \prime}=0 \\
& \tau_{3}^{\prime \prime}=\tau_{3}^{\prime}-\tau_{4}^{\prime}=I_{z z 3} \ddot{\theta}_{3}+\left(-I_{x x 3}+I_{y y 3}\right) \dot{\theta}_{2}^{2} s \theta_{3} c \theta_{3}
\end{aligned}
$$

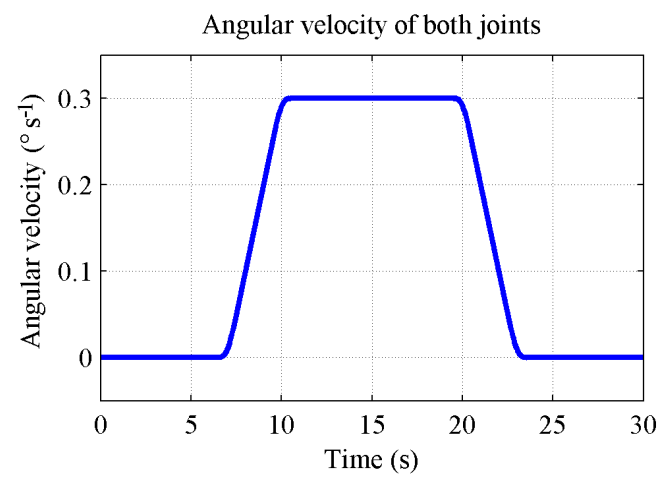

Figure 9. Angular velocity of joints.

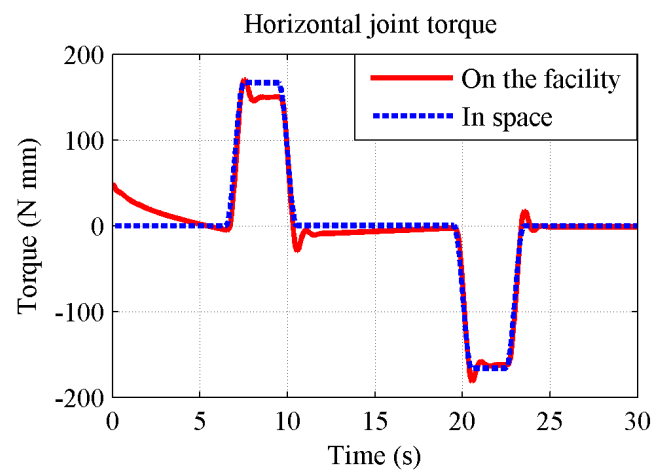

Figure 10. Horizontal joint torque.

$$
\begin{aligned}
\tau_{2}^{\prime \prime} & =\tau_{2}^{\prime}=m_{4} \ddot{\theta}_{2} l_{3}^{2}-2 m_{4} l_{3} l_{\mathrm{c} 4 z} \ddot{\theta}_{2}+m_{2} \ddot{\theta}_{2} l_{\mathrm{c} 2 y}^{2}+m_{3} \ddot{\theta}_{2} l_{\mathrm{c} 3 z}^{2} \\
& +m_{4} \ddot{\theta}_{2} l_{\mathrm{c} 4 z}^{2}+I_{y y 3} \ddot{\theta}_{2}+I_{z z 2} \ddot{\theta}_{2}\left(I_{x x 3}-I_{y y 3}\right) \ddot{\theta}_{2} s^{2} \theta_{3} \\
& +I_{y y 4} \ddot{\theta}_{2}+2\left(I_{x x 3}-I_{y y 3}\right) \dot{\theta}_{2} \dot{\theta}_{3} s \theta_{3} c \theta_{3}
\end{aligned}
$$

What's more, the whole load of the pointing mechanism is changed because of the air bearings and other parts such as the support flatbed and the test devices. So the inertia matrix of link 3 is changed in the dynamic equations.

\subsection{Effectiveness of the gravity unloading facility}

The items of torque of pointing mechanism are different between two dynamic models by comparing torque formulations. The loads of both joints are changed with the application of air bearings on the gravity unloading facility. The air spindle and planar air bearings change not only the distribution of mass but also the structure of load.

The dynamic model in space includes two rotational joints which are the joints of the APM. $\tau_{1}$ and $\tau_{2}$ are the driving torques of APM.

The dynamic model on the facility includes four joints: three rotational joints and one translational joint. The air spindle is considered as a third rotational joint which is a passive joint driven by the torque of support forces of the 


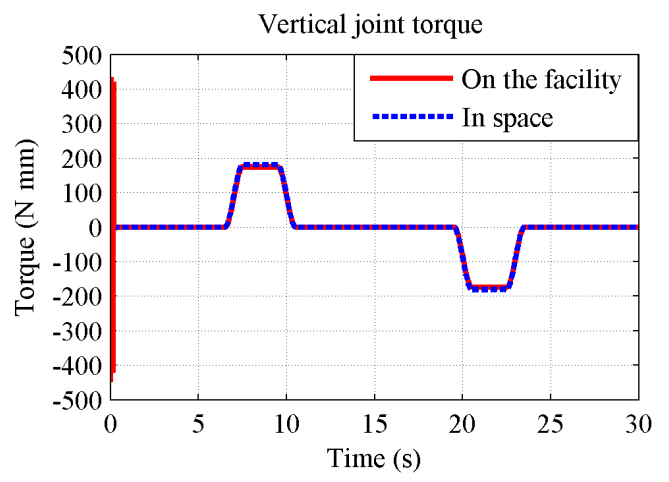

Figure 11. Vertical joint torque.

Table 4. Torque of two APM joints in both models.

\begin{tabular}{lll}
\hline & Vertical joint & Horizontal joint \\
\hline Model in space & $\tau_{1}, f_{1 \mathrm{~V}}$ & $\tau_{2}, f_{2} \mathrm{~V}$ \\
Model on facility & $\tau_{2}^{\prime \prime}, f_{2 \mathrm{~V}}^{\prime}$ & $\tau_{3}^{\prime \prime}, f_{3 \mathrm{~V}}^{\prime}$ \\
\hline
\end{tabular}

planar air bearings and the gravity. $\tau_{2}^{\prime \prime}$ and $\tau_{3}^{\prime \prime}$ are the torques of APM.

Corresponding torque of APM in tow dynamic models are shown in Table 4.

Calculate forces and torques in Table 2 with values of each item from 3-D model:

$$
\begin{aligned}
& \tau_{1}=\tau_{2}^{\prime \prime}=0.192 N \cdot m \\
& \tau_{2}=\tau_{3}^{\prime \prime}=0.185 N \cdot m \\
& f_{1 \mathrm{~V}}=f_{2 \mathrm{~V}}^{\prime}=0 \\
& f_{2 \mathrm{~V}}=f_{3 \mathrm{~V}}^{\prime}=0
\end{aligned}
$$

Though the items of both dynamic models are different, the corresponding torques and forces of APM are equal which means the running state of the APM on the facility is the same as the running state in space if the air viscous resistance is ignored. The gravity unloading facility can unload the gravity effectively.

\section{Simulation}

The 3-D model of the air bearing gravity unloading facility is designed as described in part 2, shown in Fig. 3. Another 3-D model (Fig. 6) is built to get the torque and force curves of the APM in space. Then two models are used in the simulation with ADAMS software. The angular velocity of both joints is shown in Fig. 9. Figures 10 and 11 are the torque of horizontal and vertical joints respectively in space environment and on the facility. Figures 12 and 13 are the vertical forces of horizontal and vertical joints respectively in space environment and on the facility. The angular velocity starts with 0 and then accelerates to $0.3^{\circ} \mathrm{s}^{-1}$. It decelerates to 0 again after a while. The following is angular accelerate velocity function

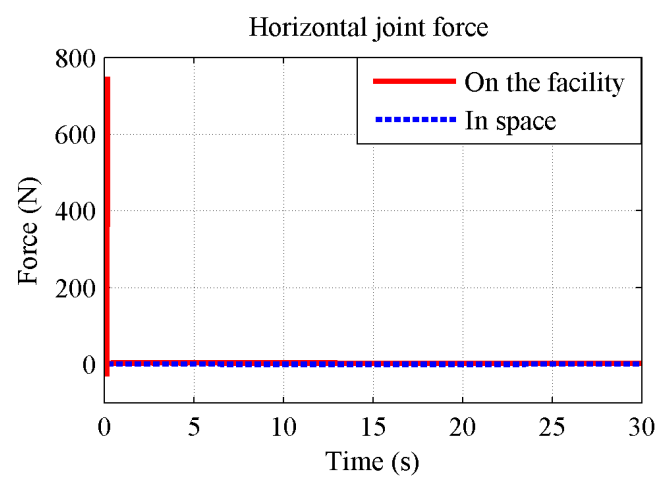

Figure 12. Horizontal joint force.

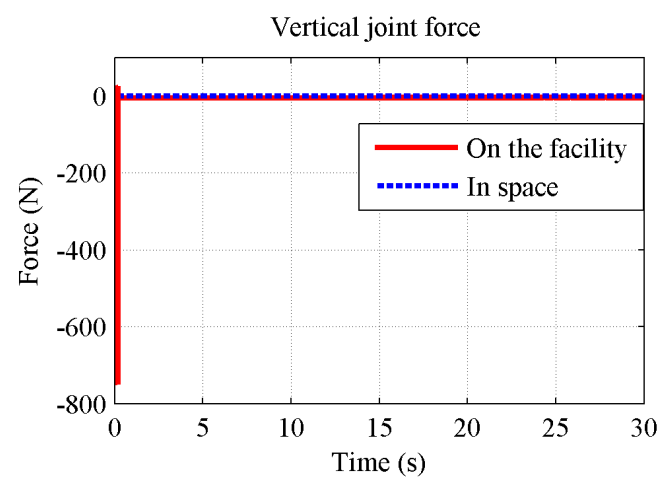

Figure 13. Vertical joint force.

in ADAMS: step(time, 6.5, 0, 7.5, 0.1d) + step(time, 9.5, 0, $10.5,-0.1 d)+\operatorname{step}($ time $, 19.5,0,20.5,-0.1 d)+$ step (time, $22.5,0,23.5,0.1 d)$.

In Fig. 10, the horizontal driving torque on the gravity unloading facility is nearly the same with the one in space environment except the first one has a slight offset down. The offset is cause by the asymmetry of the artificial load of horizontal axis especially after the application of air bearing and other connection parts. Though the balance with the counterweight is considered, the offset still exist because of the precision of software model. In the simulation, the offset can be eliminated by applying an extra force on the counterweight which means that when the real gravity unloading facility is established, the offset can be eliminated by adjusting the counterweight carefully. Besides, to imitate the fundamental modal frequency, the artificial load has a low fundamental frequency which means a low stiffness in the tangential direction of the horizontal axis. So the torque of horizontal axis has fluctuations at the acceleration and deceleration points. The fluctuations attenuate quickly, so the gravity unloading facility works stably.

In Fig. 11, the driving torque on the gravity unloading facility is the same with the one in space environment except the beginning of the vertical joint torque. On the facility, there are two reasons bring the great torque impact 

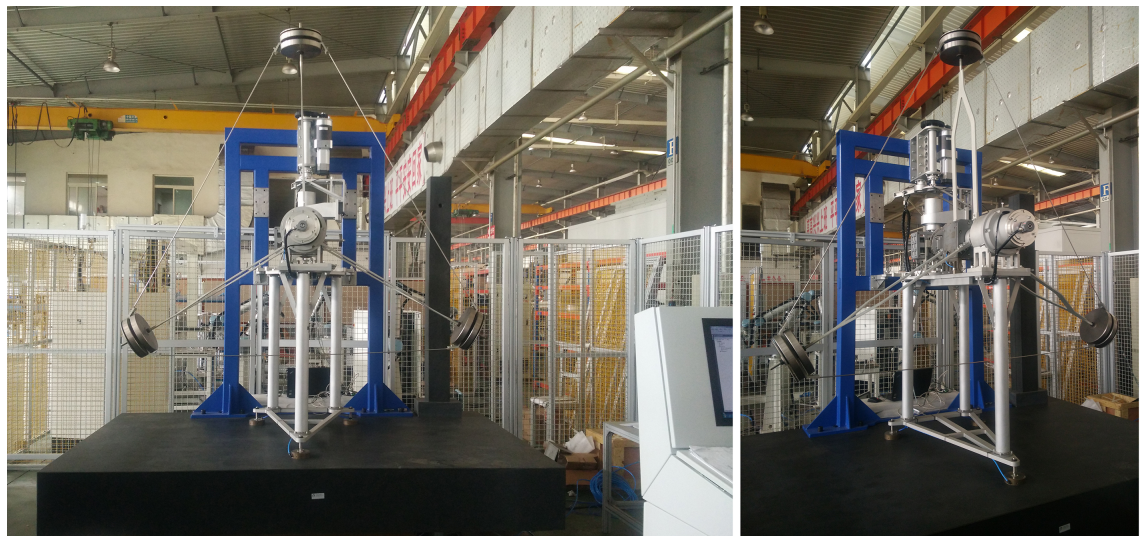

Figure 14. The facility.
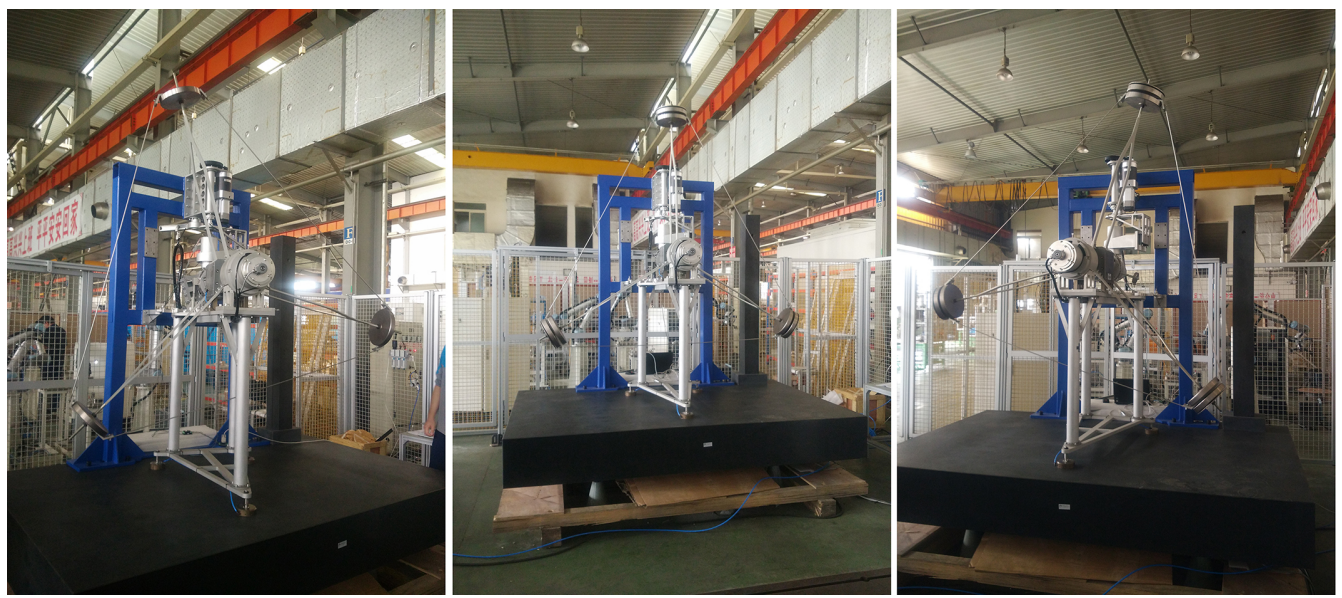

Figure 15. APM in different position on the facility.

at the beginning. Firstly, at the starting point of the whole system with the supply of high pressure air, the planar air bearings have an initial process which brings the impact of the vertical axis. Secondly, springs are used for unloading the gravity (adjustable spring mechanism-1 and 2). During the start of the simulation, there is a balance process with the spring and air bearings stiffness and damping. Compared with the torque of horizontal axis, the torque of vertical axis is smoother except the beginning. Because the stiffness in the axial direction of the artificial load is quite large, the low fundamental modal frequency has no influence on the vertical driving torque.

Figures 12 and 13 are the vertical forces of horizontal and vertical joints respectively in space environment and on the facility. The vertical forces of both joint in space are zero perfectly. Both the vertical forces of horizontal and vertical joint on the facility include an impact at the beginning with the same reason of the impact of vertical joint torque on the facility in Fig. 11. Both curves of vertical forces of horizontal and vertical joint are zero just as in space except the beginning, which means the facility can unload the gravity sufficiently.
Figures 10 to 13 show that the air bearing gravity unloading facility provides an environment of microgravity successfully. The facility can test the performance of antenna pointing mechanism effectively.

\section{Experiment}

With all the analysis and simulation done above, the gravity unloading facility is built (shown in Fig. 14) based on the method described in part 2.

The APM rotates on the gravity unloading facility freely just as it rotates in space. The following are the pictures that the joints of APM in different position (shown in Fig. 15). Before the APM is connected, the whole parts (over $150 \mathrm{~kg}$ ) on the granite platform can even be rotated or moved by only one finger which means that those parts are float by the air bearings.

There are two experiments done to illustrate the effectiveness of the gravity unloading method. The first one is to measure the thickness of the air films in the air bearings. This measurement proves that the air bearing do support the load 


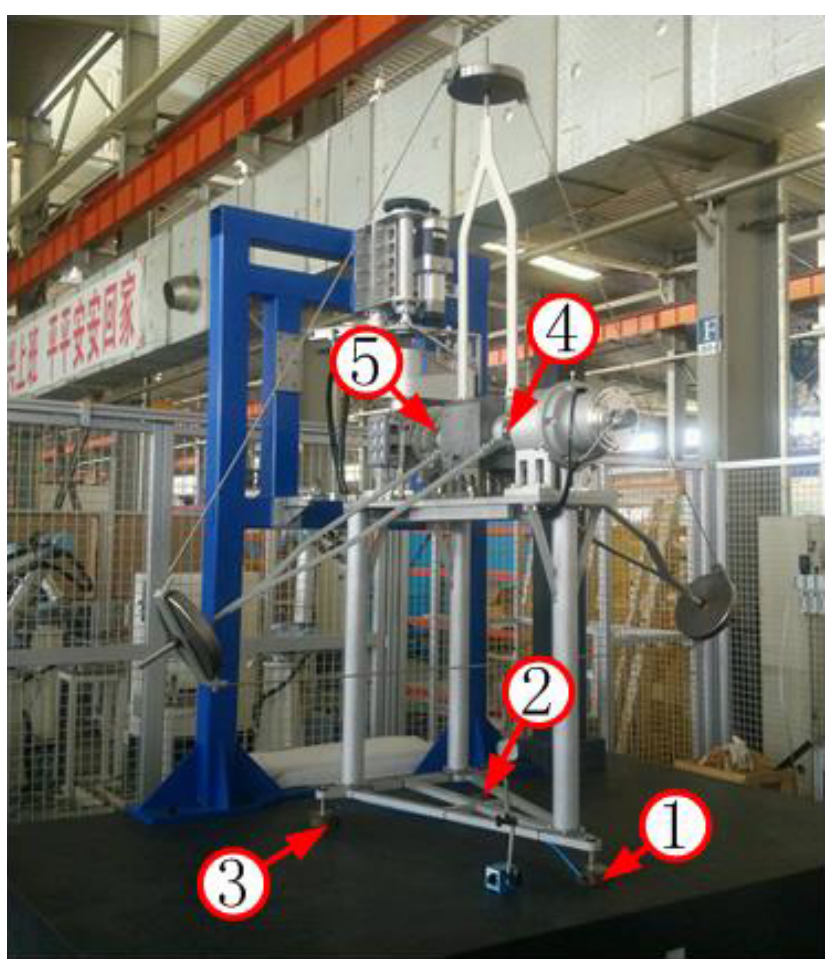

Figure 16. The points to measure the air films.

on it to unload the gravity. The second one is the measurement of the torque on the horizontal APM joint. It verifies that the load on the horizontal joint is balance and the joint is just as the state in space because it is so sensitive to the change of torque.

\subsection{Measure the thickness of the air film}

The air film in the air bearings is too thin to be seen directly when the high pressure air is connected, so the thickness of the air film must be measured by dial indicator. The air films in three planar air bearings and the air spindle are measured in 5 points on the gravity unloading facility (shown in Fig. 16). The dial indicator is put on the granite platform and adjusted to 0 before the high pressure air is supplied, then connect and disconnect the high pressure air for several times and record the thickness of air film (the dial indicator is put on the support platform to measure the air film in the air spindle).

Table 5 is the the following is the thickness of air films measured. The result shows that the air bearings are effective when the high pressure air is connected. With the thickness measured, there is high pressure air in the air bearings which means that both joints of APM rotate only with air viscous resistance which is very small and neglectable. So the APM joints rotate just as in space.
Table 5. The thickness of air films.

\begin{tabular}{|c|c|c|c|c|c|c|}
\hline \multirow[b]{2}{*}{$\begin{array}{l}\text { Measure } \\
\text { point }\end{array}$} & \multicolumn{5}{|c|}{$\begin{array}{c}\text { Times of connect and } \\
\text { disconnect the high } \\
\text { pressure air }\end{array}$} & \multirow[b]{2}{*}{$\begin{array}{r}\text { Average } \\
\text { thickness } \\
(\mu \mathrm{m})\end{array}$} \\
\hline & 1 & 2 & 3 & 4 & 5 & \\
\hline 1 & 20 & 18 & 19 & 20 & 19 & 19.2 \\
\hline 2 & 22 & 21 & 20 & 21 & 20 & 20.8 \\
\hline 3 & 22 & 22 & 21 & 20 & 21 & 21.2 \\
\hline 4 & 12 & 10 & 09 & 13 & 10 & 10.8 \\
\hline 5 & 9 & 10 & 10 & 11 & 10 & 10.0 \\
\hline
\end{tabular}

\subsection{Measure the torque of the horizontal joint}

The following figure shows the torque of horizontal joint while the high pressure air is connected and disconnected several times. Then two coins (about $3 \mathrm{~g}$ ) are pasted onto the load of horizontal joint and the torque changes accordingly, shown in the figure. This means that the horizontal joint is sensitive with the change of torque because its gravity is unloaded by the air spindle, which also means that the method is effective.

\section{Conclusion}

1. A hierarchical and simultaneous gravity unloading method with air bearings is proposed for the twoorthogonal-axis antenna pointing mechanism. The mechanism is described in detail. This method realizes the gravity unloading of the APM hierarchically and simultaneously and solves the coupling problem of two joints of APM.

2. The air bearing gravity unloading facility based on the hierarchical and simultaneous gravity unloading method is designed with planar air bearings and air spindle. The facility is a structure with two layers which compensates the gravity of both axes when they rotate one after another or together. The effectiveness of the gravity unloading method is proved by the dynamic models and simulation.

3. The dynamic models of the pointing mechanism in space environment and on the gravity unloading facility are derived respectively. Two models show that the gravity unloading facility compensates the gravity successfully after comparing the force and torque formulations. 

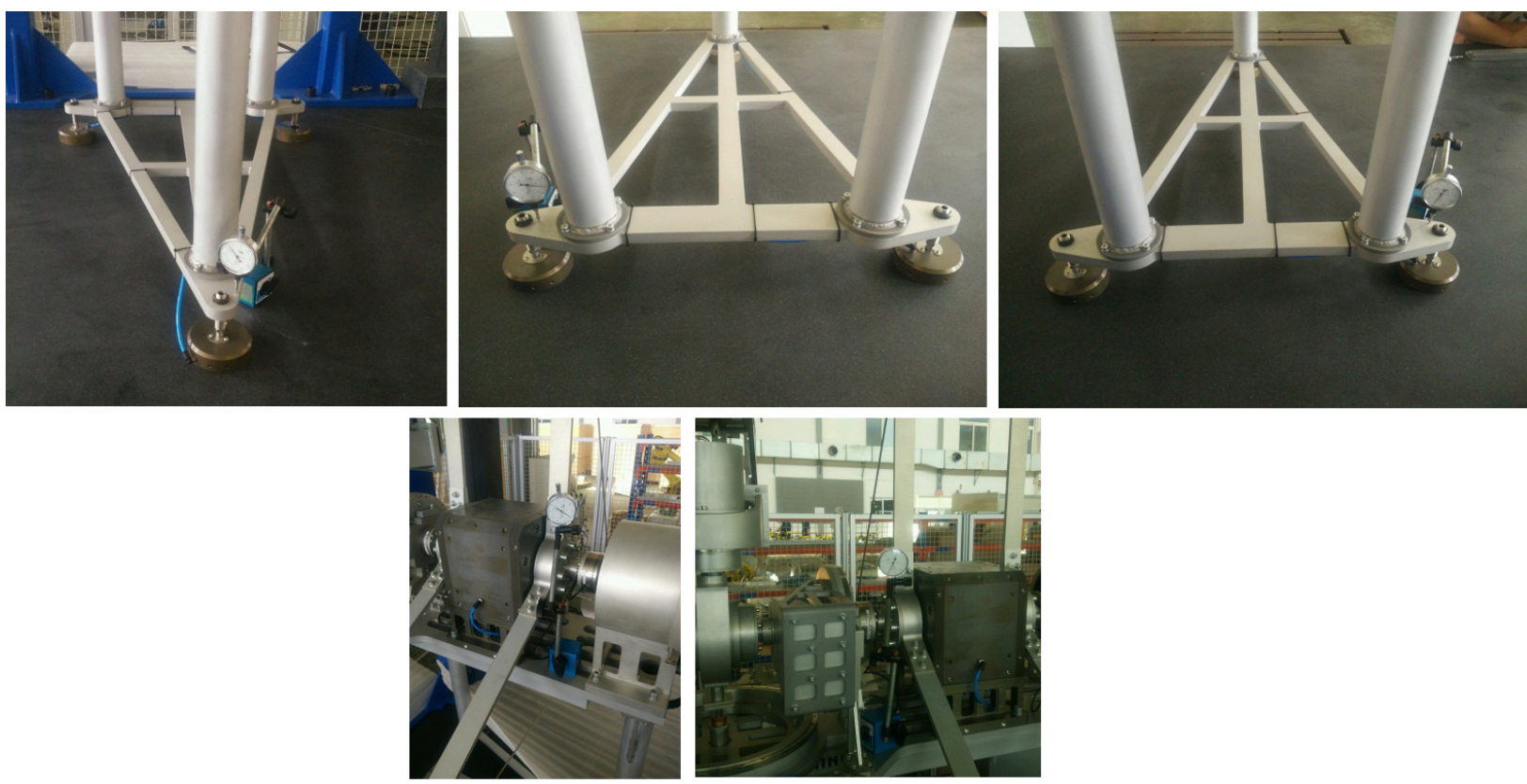

Figure 17. Measure the air films in the planar air bearing and air spindle.

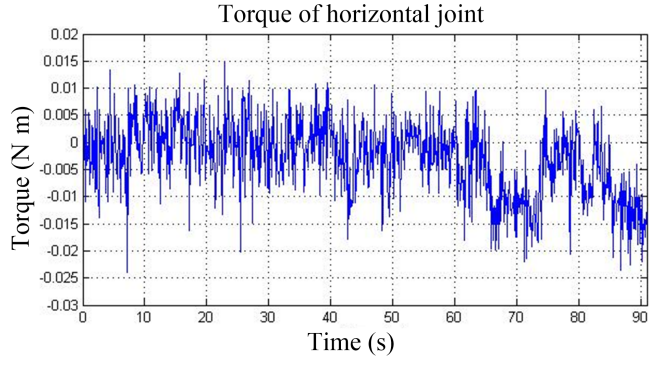

Figure 18. Torque of horizontal joint

4. The precision is analysed with the calculation of torques and vertical forces in both models. The corresponding joint torques and vertical forces of APM in both models are equal after the calculation with values from 3-D model. The result means that the gravity unloading facility unloads the gravity successfully with air bearings and the test result of APM on the facility is reliable.
5. The simulation in ADAMS also shows that the gravity unloading facility can provide microgravity environment. The asymmetry of the horizontal axis load results in the extra torque of horizontal joint. The asymmetry is brought by the structure of some connection parts which reduces the precision of the gravity unloading facility. The counterweight of the horizontal load must be adjusted carefully to eliminate the asymmetry.

6. The gravity unloading facility is established. Some experiments are done on the facility and verify the effectiveness of the facility.

Data availability. The data to this paper can be found in the Supplement. 


\section{Appendix A}

Newton-Euler method used when deriving the dynamic models.

$$
\begin{aligned}
& \text { A1 Recursive outward }(i: 0 \rightarrow n-1) \\
& { }^{i+1} \boldsymbol{\omega}_{i+1}= \begin{cases}{ }_{i}^{i+1} \mathbf{R}^{i} \omega_{i}+\dot{\theta}_{i+1}^{i+1} \mathbf{Z}_{i+1} & \text { (Rotatable joint) } \\
{ }_{i}^{i+1} \mathbf{R}^{i} \omega_{i} & \text { (Translational joint) }\end{cases}
\end{aligned}
$$

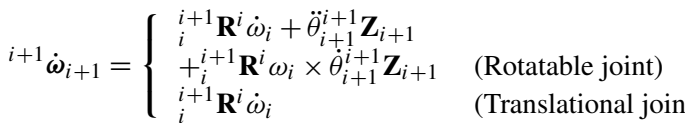

$$
\begin{aligned}
& { }^{i+1} \dot{\boldsymbol{v}}_{i+1}=\left\{\begin{array}{cc}
{ }^{i+1} \mathbf{R}\left[{ }^{i} \dot{\boldsymbol{v}}_{i}+{ }^{i} \dot{\boldsymbol{\omega}}_{i} \times{ }^{i} \boldsymbol{P}_{i+1}\right. & \\
& \left.+{ }^{i} \boldsymbol{\omega}_{i} \times\left({ }^{i} \boldsymbol{\omega}_{i} \times{ }^{i} \boldsymbol{P}_{i+1}\right)\right] \\
{ }^{i+1} \mathbf{R}\left[{ }^{i} \dot{\boldsymbol{v}}_{i}+{ }^{i} \dot{\boldsymbol{\omega}}_{i} \times \boldsymbol{P}_{i+1}\right. & \\
{ }^{i} & \left.+{ }^{i} \boldsymbol{\omega}_{i} \times\left({ }^{i} \boldsymbol{\omega}_{i} \times{ }^{i} \boldsymbol{P}_{i+1}\right)\right] \\
& +2^{i+1} \boldsymbol{\omega}_{i+1} \times \dot{d}_{i+1}^{i+1} \mathbf{Z}_{i+1} \\
+\ddot{d}_{i+1}^{i+1} \mathbf{Z}_{i+1} & \\
& \text { (Translational joint) }
\end{array}\right. \\
& { }^{i+1} \dot{\boldsymbol{v}}_{\mathrm{c} i+1}={ }^{i+1} \dot{\boldsymbol{v}}_{i+1}+{ }^{i+1} \dot{\boldsymbol{\omega}}_{i+1} \times{ }^{i+1} \boldsymbol{P}_{\mathrm{c} i+1} \\
& +{ }^{i+1} \boldsymbol{\omega}_{i+1} \times\left({ }^{i+1} \boldsymbol{\omega}_{i+1} \times{ }^{i+1} \boldsymbol{P}_{\mathrm{c} i+1}\right) \\
& { }^{i+1} \boldsymbol{F}_{i+1}=m_{i+1}^{i+1} \dot{\boldsymbol{v}}_{\mathrm{c} i+1} \\
& { }^{i+1} \boldsymbol{N}_{i+1}={ }^{\mathrm{c} i+1} \mathbf{I}_{i+1}^{i+1} \dot{\boldsymbol{\omega}}_{i+1}+{ }^{i+1} \boldsymbol{\omega}_{i+1} \\
& \times\left({ }^{\mathrm{c} i+1} \mathbf{I}_{i+1}^{i+1} \boldsymbol{\omega}_{i+1}\right)
\end{aligned}
$$

\section{A2 Recursive inward $(i: n \rightarrow 1)$}

${ }^{i} \boldsymbol{f}_{i}={ }_{i+1}^{i} \mathbf{R}^{i+1} \boldsymbol{f}_{i+1}+{ }^{i} \boldsymbol{F}_{i}$

${ }^{i} \boldsymbol{n}_{i}={ }_{i+1}^{i} \mathbf{R}^{i+1} \boldsymbol{n}_{i+1}+{ }^{i} \boldsymbol{N}_{i}+{ }^{i} \boldsymbol{P}_{\mathrm{c} i} \times{ }^{i} \boldsymbol{F}_{i}$

$$
+{ }^{i} \boldsymbol{P}_{i+1} \times{ }_{i+1}^{i} \mathbf{R}^{i+1} f_{i+1}
$$

$\tau_{i}=\left\{\begin{array}{cl}{ }^{i} \boldsymbol{n}_{i}^{T^{i}} \mathbf{Z}_{i} & \text { (Rotatable joint) } \\ { }^{i} \boldsymbol{f}_{i}^{T^{i}} \mathbf{Z}_{i} & \text { (Translational joint) }\end{array}\right.$

where ${ }^{i} \omega_{i}$ is the angular velocity of link $i$ in coordinate $i$ while ${ }^{i} \dot{\boldsymbol{\omega}}_{i}$ is the angular acceleration, $\dot{\theta}_{i}$ is the angle velocity of joint $i, \ddot{\theta}_{i}$ is the angle acceleration of joint $i,{ }_{i+1}^{i} \mathbf{R}$ is the transformation matrix from coordinate $i+1$ to coordinate $i$, ${ }^{i} \dot{\boldsymbol{v}}_{i}$ is the acceleration of the origin point of the coordinate system $i$ while ${ }^{i} \dot{\boldsymbol{v}}_{\mathrm{c} i}$ is the acceleration of gravity center of link $i,{ }^{i} \boldsymbol{P}_{i+1}$ is the vector of coordinate $i+1$ origin point in coordinate $i, \dot{d}_{i}$ is the velocity of translational joint $i,{ }^{i} P_{\mathrm{C} i}$ is the vector of gravity center of link $i,{ }^{i} \boldsymbol{F}_{i}$ and ${ }^{i} \boldsymbol{N}_{i}$ are the inertial force and torque of link $i, m_{i}$ is the mass of link $i,{ }^{\mathrm{C} i} \mathbf{I}_{i}$ is the inertia matrix of link $i$ through gravity center with an expression of $\operatorname{diag}\left(I_{x x i}, I_{y y i}, I_{z z i}\right) .{ }^{i} \boldsymbol{f}_{i}$ and ${ }^{i} \boldsymbol{n}_{i}$ are the force and torque of joint $i, \tau_{i}$ is the torque of joint $i$.

\section{Appendix B}

The forces in model of Fig. 7:

$\begin{aligned}{ }^{1} \boldsymbol{f}_{1}= & {\left[\begin{array}{c}m_{2} \ddot{\theta}_{1} l_{\mathrm{c} 2}-m_{1} \ddot{\theta}_{1} l_{\mathrm{c} 1 y} \\ m_{2} \dot{\theta}_{1}^{2} l_{\mathrm{c} 2}-m_{1} \dot{\theta}_{1}^{2} l_{\mathrm{c} 1 y} \\ 0\end{array}\right] } \\ { }^{2} f_{2}= & {\left[\begin{array}{c}m_{2} \ddot{\theta}_{1} l_{\mathrm{c} 2} c \theta_{2} \\ -m_{2} \ddot{\theta}_{1} l_{\mathrm{c} 2} s \theta_{2} \\ -m_{2} \dot{\theta}_{1}^{2} l_{\mathrm{c} 2}\end{array}\right] }\end{aligned}$

The forces in model of Fig. 8:

$$
\begin{aligned}
{ }^{2} \boldsymbol{f}_{2}^{\prime} & =\left[\begin{array}{r}
f_{2 x} \\
f_{2 y} \\
f_{2 z}
\end{array}\right] \\
{ }^{3} \boldsymbol{f}_{3}^{\prime} & =\left[\begin{array}{r}
f_{3 x} \\
f_{3 y} \\
f_{3 z}
\end{array}\right] \\
f_{2 x} & =\ddot{\theta}_{2}\left(l_{3} m_{4}-l_{\mathrm{c} 2 y} m_{2}+l_{\mathrm{c} 3 z} m_{3}-l_{\mathrm{c} 4 z} m_{4}\right) \\
f_{2 y} & =l_{\mathrm{c} 3 z} m_{3} \dot{\theta}_{2}^{2}-l_{\mathrm{c} 2 y} m_{2} \dot{\theta}_{2}^{2}+m_{4} \dot{\theta}_{2}^{2}\left(l_{3}-l_{\mathrm{c} 4 z}\right) \\
f_{2 z} & =F_{\mathrm{e} 41}+F_{\mathrm{e} 42}+F_{\mathrm{e} 43}+\left(m_{2}+m_{3}+m_{4}\right)\left(\ddot{d}_{1}-g\right) \\
f_{3 x} & =M_{1} \sin \theta_{3}+m_{3} M_{2}+m_{4} \ddot{\theta}_{2} \cos \theta_{3}\left(l_{3}-l_{\mathrm{c} 4 z}\right) \\
f_{3 y} & =M_{1} \cos \theta_{3}-m_{3} M_{3}-m_{4} \ddot{\theta}_{2} \sin \theta_{3}\left(l_{3}-l_{\mathrm{c} 4 z}\right) \\
f_{3 z} & =-l_{\mathrm{c} 3 z} m_{3} \dot{\theta}_{2}^{2}-m_{4} \dot{\theta}_{2}^{2}\left(l_{3}-l_{\mathrm{c} 4 z}\right) \\
M_{1} & =F_{\mathrm{e} 41}+F_{\mathrm{e} 42}+F_{\mathrm{e} 43}-k_{2} \Delta x_{2}+m_{4}\left(\ddot{d}_{1}-g\right) \\
M_{2} & =\ddot{d}_{1} \sin \theta_{3}-g \sin \theta_{3}+l_{\mathrm{c} 3 z} \ddot{\theta}_{2} \cos \theta_{3} \\
M_{3} & =g \cos \theta_{3}-\ddot{d}_{1} \cos \theta_{3}+l_{\mathrm{c} 3 z} \ddot{\theta}_{2} \sin \theta_{3}
\end{aligned}
$$

where ${ }^{1} \boldsymbol{f}_{1}$ and ${ }^{2} \boldsymbol{f}_{2}$ are the forces of vertical and horizontal joints of model in space respectively, ${ }^{2} \boldsymbol{f}_{2}^{\prime}$ and ${ }^{3} \boldsymbol{f}_{3}^{\prime}$ are the forces of vertical and horizontal joints of model on the gravity unloading facility respectively. $\dot{\theta}_{i}$ is the angle velocity of joint $i, \ddot{\theta}_{i}$ is the angle acceleration of joint $i, m_{i}$ is the mass of link $i,\left(0,0, l_{\mathrm{c} 2}\right)^{\mathrm{T}}$ is the gravity center position of link 2 in $O X_{2} Y_{2} Z_{2}\left(l_{\mathrm{c} 2}\right.$ is special in Fig. 7 while the definitions of other symbol are applicable for both model $),\left(0, l_{\text {ciy }},-l_{\text {ciz }}\right)^{\mathrm{T}}$ is the gravity center position of link $i$ in coordinate $i . F_{\mathrm{e} 41}$, $F_{\mathrm{e} 42}$ and $F_{\mathrm{e} 43}$ are the unloading forces of planar air bearings acting on link $4, k_{2}$ is the stiffness of the adjustable spring mechanism-1 while the $\Delta x_{2}$ is the deformation of the spring, $\ddot{d}_{1}$ is the acceleration of translational joint $1, g$ is the gravity acceleration, Joint 1 is static when the facility is running stable, so $\ddot{d}_{1}$ is 0 . Besides, three planar air bearings support $m_{2}, m_{3}$ and $m_{4}$ while adjustable spring mechanism-1 supports $m_{2}$. So $F_{\mathrm{e} 41}+F_{\mathrm{e} 42}+F_{\mathrm{e} 43}=\left(m_{2}+m_{3}+m_{4}\right) g$ and $m_{2} g=k_{2} \Delta x_{2}$. 


\section{The Supplement related to this article is available online at doi:10.5194/ms-8-51-2017-supplement.}

Competing interests. The authors declare that they have no conflict of interest.

Acknowledgements. This study was co-supported by the State Key Laboratory of Robotics, Shenyang Institute of Automation Chinese Academy of Sciences and Beijing Institute of Control Engineering. This paper is supported by the project of ControlCapture Integrated Test System (0002546159001).

Edited by: L. Romdhane

Reviewed by: Z. Affi and one anonymous referee

\section{References}

Atkins, E. M., Lennon, J. A., and Peasco, R. S.: Vision-based following for cooperative astronaut-robot operations, IEEE Proceedings Aerospace Conference, Big Sky, MT, USA, 2002.

Chernesky, V. S.: Development and control of a three-axis satellite simulator for the bifocal relay mirror initiative, DTIC Document, Naval Postgraduate School Publisher Location, Monterey, California, USA, 2001

Chung, S.-J.: Nonlinear control and synchronization of multiple Lagrangian systems with application to tethered formation flight spacecraft, Massachusetts Institute of Technology, 2007.

Hal, J. S. C.: Comparison of system-identification techniques for a spherical air-bearing spacecraft simulator, AIAA/AAS Astrodynamics 2003 Conference, BIG SKY, MT, 3-7 August 2003, Simulation Method of Space Operation on the Ground, 1725-1741, 2003.

Jagannathan, S., Fenn, R. C., and Johnson, B. G.: Low-cost active anti-gravity suspension system, Proceedings of American Control Conference, Seattle, WA, USA, 1995.

Jinpeng, Y., Di, Y., and Xiaosong, S.: Single access antenna pointing control system design of TDRS, 1st International Symposium on Systems and Control in Aerospace and Astronautics, ISSCAA, Harbin Inst. of Tech. Harbin, China, 2006.

Jung, D. and Tsiotras, P.: An experimental validation of spacecraft attitude and power tracking with variable speed control moment gyroscopes, AAS Spaceflight Mechanics Meeting, Sedona, AZ, 2007.

Qi, N. M., Zhang, W. H., and Gao, J. Z.: The Primary Discussion for the Ground Simulation System of Spatial Microgravity, Aerospace Control, 29, 95-100, 2011.

Sandor, V., Nick, L., and Stephen, G.: Testbed for Satellite Formation Flying Control System Verification, AIAA Infotech@Aerospace 2007 Conference and Exhibit, American Institute of Aeronautics and Astronautics, Rohnert Park, California, USA, 2007.
Sato, N. and Wakabayashi, Y.: JEMRMS design features and topics from testing, 6th International symposium on artificial intelligence, robotics and automation in space (iSAIRAS), Quebec, 2001.

Scharf, D. P., Keim, J. A., and Hadaegh, F. Y.: Flight-Like Ground Demonstrations of Precision Maneuvers for Spacecraft Formations - Part II, IEEE Systems Journal, 4, 96-106, 2010.

Schwartz, J. L. and Hall, C. D.: System identification of a spherical air-bearing spacecraft simulator, AAS Paper, 122, 2004.

Shaw, H. C., McLaughlin, B., Stocklin, F., Fortin, A., Israel, D., Dissanayake, A., Gilliand, D., LaFontaine, R., Broomandan, R., and Hyunh, N.: Applying a space-based security recovery scheme for critical homeland security cyberinfrastructure utilizing the NASA Tracking and Data Relay (TDRS) based Space Network, 2015 IEEE International Symposium onTechnologies for Homeland Security (HST), 2015.

Umetani, Y. and Yoshida, K.: Experimental study on twodimensional free-flying robot satellite model, JPL, California Inst. of Tech, Proceedings of the NASA Conference on Space Telerobotics, 1989.

Xu, J.: Prototype Development and Research on Key Technologies of The Five Degrees-of-freedom Air Bearing Spacecraft Simulator, Harbin Institute of Technology, 2010.

Yangsheng, X., Brown, B., Aoki, S., and Kanade, T.: Mobility And Manipulation Of A Light-weight Space Robot, Proceedings of the 1992 IEEE/RSJ International Conference on Intelligent Robots and Systems, 1992.

Yao, Y. S. and Mei, T.: Simulation Method of Space Operation on the Ground-Buoyancy Method, Chin. J. Mech. Eng., 182-188, 2008.

Zhai, K. and Baoyin, H.: Study of zenith pass problem of the intersatellite linkage antenna, 10th International Conference on Control, Automation, Robotics and Vision, ICARCV, Melia Hanoi, Vietnam, 2008.

Zhang, S. J. and Cao, X. B.: Physical Simulation System for Spacecraft Rendezvous and Docking Based on MicroSim, Aerospace Control, 2, 63-67, 2006.

Zheng, Y. J., Zhang, D. Z., and Chen, Y.: Research on Credibility of Air-Bearing Table-Based Physical Simulation System for Space Robot, Aerospace Control and Application, 36, 33-38, 2010.

Zhu, Z. X. and Yuan, J. P.: Microgravity environment Construction of Spacecraft operations, China Astronautic Publishing House, Beijing, 111-150, 2013.

Zou, F.: TDRS based navigation system for flight vehicles in near space, IEEE International Conference on Signal Processing, Communications and Computing (ICSPCC), Xi'an, 2011. 